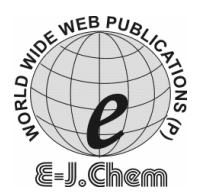

http://www.e-journals.net
ISSN: 0973-4945; CODEN ECJHAO

E-Journal of Chemistry

Vol. 5, No.3, pp. 539-550, July 2008

\title{
Investigation of Electrochemical Behaviour of Quercetin on the Modified Electrode Surfaces with Procaine and Aminophenyl in Non-Aquous Medium
}

\author{
IBRAHIM ENDER MULAZIMOGLU* and ERDAL OZKAN \\ Selçuk University, Department of Chemistry, 42099, Konya, Turkey \\ mulazimoglu@gmail.com
}

Received 2 January 2008; Revised 2 February 2008; Accepted 1 March 2008

\begin{abstract}
In this study, cyclic voltammetry and electrochemical impedance spectroscopy have been used to investigate the electrochemical behaviour of quercetin (3,3',4',5,7-pentahydroxyflavone) on the procaine and aminophenyl modified electrode. The modification of procaine and aminophenyl binded electrode surface with quercetin was performed in $+0,3 /+2,8 \mathrm{~V}$ (for procaine) and $+0,4 /+1,5 \mathrm{~V}$ (for aminophenyl) potential range using $100 \mathrm{mV} \mathrm{s}^{-1}$ scanning rate having 10 cycle. A solution of $0.1 \mathrm{M}$ tetrabutylammonium tetrafluoroborate in acetonitrile was used as a non-aquous solvent. For the modification process a solution of $1 \mathrm{mM}$ quercetin in $0.1 \mathrm{M}$ tetrabutylammonium tetrafluoroborate was used. In order to obtain these two surface, a solution of $1 \mathrm{mM}$ procaine and $1 \mathrm{mM}$ nitrophenyl diazonium salt in $0.1 \mathrm{M}$ tetrabutylammonium tetrafluoroborate was used. By using these solutions bare glassy carbon electrode surface was modified. Nitrophenyl was reduced to amine group in $0.1 \mathrm{M} \mathrm{HCl}$ medium on the nitrophenyl modified glassy carbon elelctrode surface. Procaine modified glassy carbon electrode surface was quite electroactive. Although nitrophenyl modified glassy carbon elelctrode surface was electroinactive, it was activated by reducing nitro group into amine group. For the characterization of the modified surface 1 $\mathrm{mM}$ ferrocene in $0.1 \mathrm{M}$ tetrabutylammonium tetrafluoroborate for cyclic voltammetry and $1 \mathrm{mM}$ ferricyanide/ferrocyanide (1:1) mixture in $0,1 \mathrm{M} \mathrm{KCl}$ for electrochemical impedance spectroscopy were used.
\end{abstract}

Keywords: Quercetin, Cyclic Voltammetry, Nitrophenyl diazonium salt,

\section{Introduction}

Polyphenols are complex substances with two or more phenolic rings joined together. Polyphenolic compounds can be classified depending on their chemical structure ${ }^{1}$. Polyphenols, flavonoids, and tannins are widely distributed in plants, mainly in fruits and vegetables, contributing to their flavor and color $^{2}$. 
The flavonoids constitute a large class of compounds containing a number of phenolic hydroxyl groups attached to ring structures, conferring antioxydant activity ${ }^{3-6}$. It has been shown that the antioxydant activity of flavonoids is due to the aromatic - $\mathrm{OH}$ groups ${ }^{7}$. The structural groups undergoing electron transfer reactions in the flavonoids are: (1) pyrogallol group, (2) catechol group, (3) 2,3-double bond in conjugation with a 4-oxo group and a 3-hydroxyl group and (4) additional resonance-effective substituents ${ }^{8}$. Electrochemical measurements leading to the determination of physicochemical parameters for antioxydants (e.g., redox potential, number of electrons transferred, electrodereaction rate constant, etc), are very relevant not only for evaluating the antioxidative abilities of flavonoids ${ }^{9}$ but also for understanding their reaction mechanisms. The half-wave potential $\left(\mathrm{E}_{1 / 2}\right)$ is a useful parameter for providing information on the antioxydant activity of the flavonoids. This has been rationalized on the basis that both electrochemical oxidation and hydrogen-donating free radical scavenging involve the breaking of the same phenolic bond between oxygen and hydrogen, producing the phenoxy radical and hydrogen, in an electron and proton transfer reaction. Thus a flavonoid which has a low value of $\mathrm{E}_{1 / 2}$ is a good antioxydant ${ }^{2}$. The mechanism of oxidation of phenols is of interest particularly in connection with biosynthetic-type oxidative coupling reactions. Oxidation can occur either in a single one-electron, single two-electron or in two one-electron steps ${ }^{10,11}$. Flavonoids are among widely distributed secondary products found in many plants used as food ${ }^{12-13}$ Several of these biological effects are related to radical antioxydant properties of such compounds ${ }^{13-15}$. Radical reactions occur in many biological processes as a natural consequence of living in an oxidizing environment. However damaging radical reactions outcome when an unusual process takes place $^{15}$. The antioxydant properties of these compounds (flavonoids) have been attributed to their capacity to antioxydant free radicals generated in aquous phase, increasing the resistance of lipids against peroxidation ${ }^{16}$. Despite this fact, the electrochemical behavior of quercetin has been almost always studied in organic solvents due to constrains imposed by its solubility characteristics. Evidently, the stability of intermediate species originated by oxidation reactions is substantially different depending on the surroundings (organic or aquous media) ${ }^{17}$, offering a very rich chemistry as a result. In this framework, the knowledge of its electrochemical behavior in biological-like conditions is relevant to get in sight into the quercetin action as antioxydant. To date, few information on the electrochemical properties of flavonoids in aquous solutions are found in the literature ${ }^{18}$ The electrochemistry of quercetin, in particular, besides not being very understood, reveals contradictions on both the oxidation products and the reversibility of the reactions. For instance, a limited extent of data available in the related literature suggests that the oxidation of quercetin yields to the corresponding ortho-quinone as product, which is an unstable compound that could undergo homogeneous chemical reactions such as intramolecular rearrangements ${ }^{18-21}$ or addition reactions when in presence of nucleophiles ${ }^{22}$. Therefore, the presence of water and/or ethanol (nucleophiles) in the solution may thus have very important implications for the oxidation mechanism and in turn for the antioxydant activity, since water was found acting as catalysts in reactions subsequent to the formation of ortho-quinones ${ }^{23}$.

Quercetin (3, 3', 4', 5, 7-pentahydroxyflavone-Scheme 1) is widely distributed in the plant kingdom and is the most abundant of the flavonoid molecules. Quercetin is the aglycone (i.e., minus the sugar group) of a number of other flavonoids, including rutin, quercetrin, isoquercetin, and hyperoside. These molecules have the same structure asquercetin except that they have a specific sugar molecule in place of one of quercetin is hydroxyl groups on the $\mathrm{C}$ ring, which sharply changes the activity of the molecule. Activity comparison studies have identified other flavonoids as often having similar effects as quercetin; but quercetin usually has the greatest activity. Quercetin appears to cause many beneficial effects on human health, including cardiovascular protection, anticancer activity, anti-ulcer effects, anti-allergy activity, 
cataract prevention, antiviral activity, and anti-inflammatory effects ${ }^{21}$. Quercetin is a flavonoid of widespread existence in nature whose medicinal properties have been extensively demonstrated in the literature, especially the antioxydant capacity ${ }^{16,23-26}$. Solubility of quercetin is low in water, but it generally dissolves remarkably in organic solvent (acetonitrile, alcohol, etc). The flavonoids present in tomatoes, tobaccos, rue and in numerous vegetables, fruits and grains. Its chemical structure contains the resorcinol group in the ring $\mathrm{A}$ (with the m-hydroxyls in the positions $\mathrm{C} 5$ and $\mathrm{C} 7$ ), the catechol group in the ring B (with the o-hydroxyls in the positions C3' and C4') and the hydroxyl group in the position $\mathrm{C} 3$ of the ring $\mathrm{C}$, the carbonyl group in the position $\mathrm{C} 4$ in the ring $\mathrm{C}$ and the $\mathrm{C}=\mathrm{C}$ bond between the carbons $\mathrm{C} 2$ and $\mathrm{C} 3$ in the ring $\mathrm{C}$. As can be concluded, quercetin presents $\mathrm{OH}$ functional groups that can be electrochemically oxidized. The oxidation of the 3', 4' dihydroxy group in the ring B can lead to the formation of the corresponding o-quinone. Unlike, the 5, 7 dihydroxy group in the ring A cannot lead to the formation of the corresponding m-quinone. Additionally, the catechol group in the ring $\mathrm{B}$ is oxidized more easily than the resorcinol group in the ring $\mathrm{A}^{27}$.<smiles></smiles>

\section{Experimental}

Scheme 1. The molecular structure of quercetin

Ultra pure quality of water with a resistance of $18.3 \mathrm{M} \Omega \mathrm{cm}$ (Millipore Milli-Q purification system, Millipore Corp. Bedford, MA, USA) was used in preparations of aquous solutions, cleaning of the glassware and polishing the electrodes. All chemicals were of the highest purity available from Merck (Darmstadt, Germany), Fluka (Buchs SG, Switzerland), Riedelde Haën (Seelze, Germany) or Sigma-Aldrich (Buchs SG, Switzerland), chemical companies and so no further purification was performed. nitrophenyl diazonium salt (NPDAS) was synthesized from $p$-nitroaniline (Aldrich). In all experiments, the solutions and the electrodes were kept in acetonitrile when they were not in use. All the experiment solutions were prepared at $1 \mathrm{mM}$ concentration. Solutions were thoroughly deoxygenated by purging with purified argon gas (99.99\%) for 10 min prior to the electrochemical experiments. Argon blanket was maintained over the solutions to supply an inert atmosphere during voltammetric measurements. All electrochemical experiments were performed at room temperature $\left(25 \pm 1^{\circ} \mathrm{C}\right)$.

\section{Electrodes and apparatus}

A traditional three-electrode cell system was used in all electrochemical experiments. In our experiments $\mathrm{Ag} / \mathrm{Ag}^{+}(0.01 \mathrm{M})$, reference electrode calibrated to the $E_{1 / 2}$ of ferrocene and a $\mathrm{Pt}$ wire counter electrode was employed. BAS (Bioanalytical Systems) Model MF-2012 and Tokai GC-20 glassy carbon electrodes were used as working electrodes having a surface of $0.071 \mathrm{~cm}^{2}$. CV and EIS techniques were performed using GAMRY reference 750 potentiostat/galvanostat/ZRA with C3 cell stand (BAS). 


\section{Procedure}

\section{Preparation of the electrodes}

The glassy carbon electrodes (GCE) were prepared for the experiments by polishing to gain a mirror-like appearance, first with fine wet emery papers (grain size 4000) and then with $1.0 \mu \mathrm{m}$ and $0.3 \mu \mathrm{m}$ alumina slurry on micro cloth pads (Buehler, USA). After the initial polishing, the GCE electrodes were resurfaced using $0.05 \mu \mathrm{m}$ alumina slurry. First, the GCE electrodes were sonicated in the water twice then in 1:1 (v/v) isopropyl alcohol and acetonitrile (IPA $+\mathrm{MeCN})\left(\right.$ Aldrich) mixture for $10 \mathrm{~min}$. each ${ }^{28}$.

\section{Synthesis of NPDAS}

Approximately $0.5 \mathrm{~g}$ of amino precursor $p$-nitroaniline (Aldrich) was weighed into a three-necked $50 \mathrm{~mL}$ round-bottom flask; then $10 \mathrm{~mL}$ of $50 \%$ fluoroboric acid (Aldrich) was added and the resultant mixture was stirred with a magnetic stirring bar, for an hour at $10^{\circ} \mathrm{C}$. A 5:1 molar ratio of $\mathrm{NaNO}_{2}$ (relative to amino precursor) was weighed into a separate container, just enough water was added to dissolve the $\mathrm{NaNO}_{2}$ at room temperature, and the solution was cooled to $10^{\circ} \mathrm{C}$. A thermometer was inserted into the three-necked flask, and the precursor solution was cooled to $10{ }^{\circ} \mathrm{C}$ in the ice bath. The cold $\mathrm{NaNO}_{2}$ solution was added dropwise, and the temperature was always kept below $10^{\circ} \mathrm{C}$ during the reaction. Following the complete addition of $\mathrm{NaNO}_{2}$, the mixture was stirred $\approx 30 \mathrm{~min}$. in the ice bath. The insoluble diazonium salt was filtered in a Buchner funnel, and anhydrous ether (Fluka) was used to remove the remaining sediments from the round-bottom flask. The product was recrystallized by dissolving in cold $\left(+4^{\circ} \mathrm{C}\right)$ acetonitrile followed by slow addition of cold anhydrous ether to recover the diazonium tetrafluoroborate salt (Scheme 2). The synthesized DAS should be kept in a freezer and should be used within two mounts. Otherwise DAS may decompose. It one wants to use the decomposed DAS, one should recrystallise it. DAS should be prepared freshly and used immediately within two hours of preparation ${ }^{29,30}$

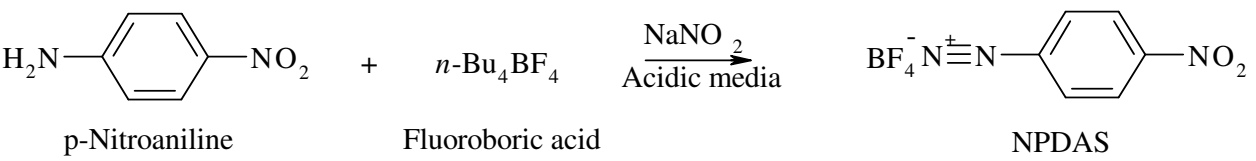

Scheme 2. Reaction mechanism of nitrophenyl diazonium salt (NPDAS) synthesis

\section{Results and Discussion}

This study has been performed using cyclic voltammetry (CV) and electrochemical impedance spectroscopy (EIS) to investigate the electrochemical behaviour of quercetin $(\mathrm{QR})$ on the procaine (PR) and aminophenyl (AP) modified (GCE) surfaces in non-aquous medium. From the data obtained we examined the electrochemical and spectroscpic behaviour of the electrode surface. Depending on the literature we suggested a reaction mechanism.

\section{Modification and Characterization of Quercetin on PR-GC}

In order to investigate the electrochemical behaviour of QR on the PR modified GCE (PR-GCE) surface, $1 \mathrm{mM}$ PR solution in $0.1 \mathrm{M}$ TBATFB was used. The potential range was $-1.7 /-0.5 \mathrm{~V}$ using $100 \mathrm{mV} \mathrm{s}^{-1}$ scanning rate with 50 cycle and given in Figure1. 


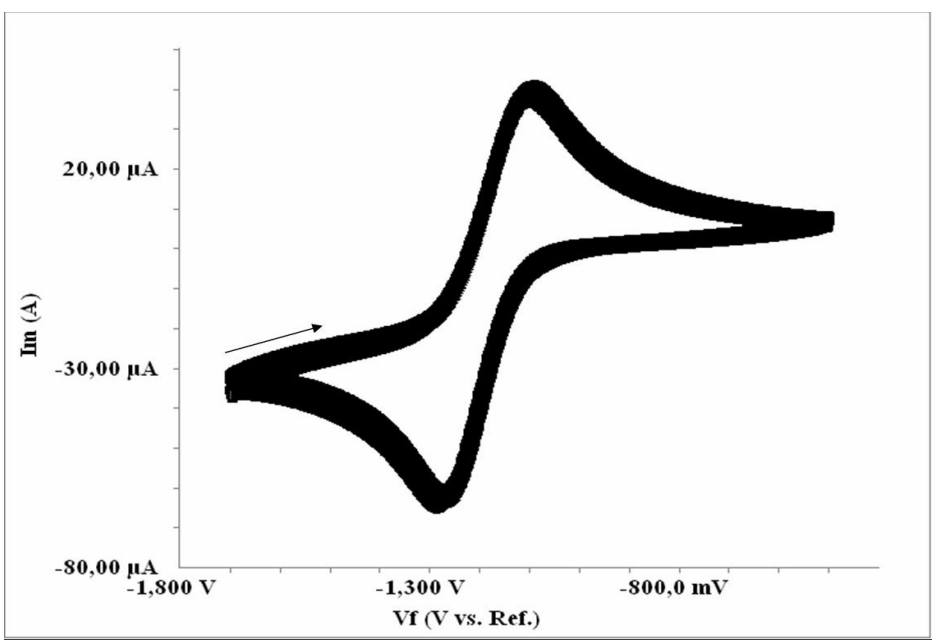

Figure 1. Cyclic voltammograms of procaine on bare glassy carbon electrode surface, -1,7/$0,5 \mathrm{~V}$ potential range $100 \mathrm{mV} \mathrm{s}^{-1}$ scanning rate having 50 cycle

The reaction mechanism for the modification process is given in Scheme 3. we found that PR-GCE surface more active than bare GCE surface by testing the surface by CV and EIS.

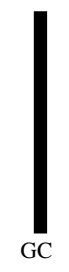<smiles>CCN(CC)CCOC(=O)c1ccc(N)cc1</smiles><smiles>CCN(CC)CCOC(=O)c1ccc(N)cc1</smiles>

Scheme 3. Modification of procaine hydrochloride on bare GCE

The modification of QR on the obtained PR-GCE surface was carried out in $+0.3 /+2.8 \mathrm{~V}$ potential range using $100 \mathrm{mV} \mathrm{s}^{-1}$ scanning rate with 10 cycle and given in Figure 2.

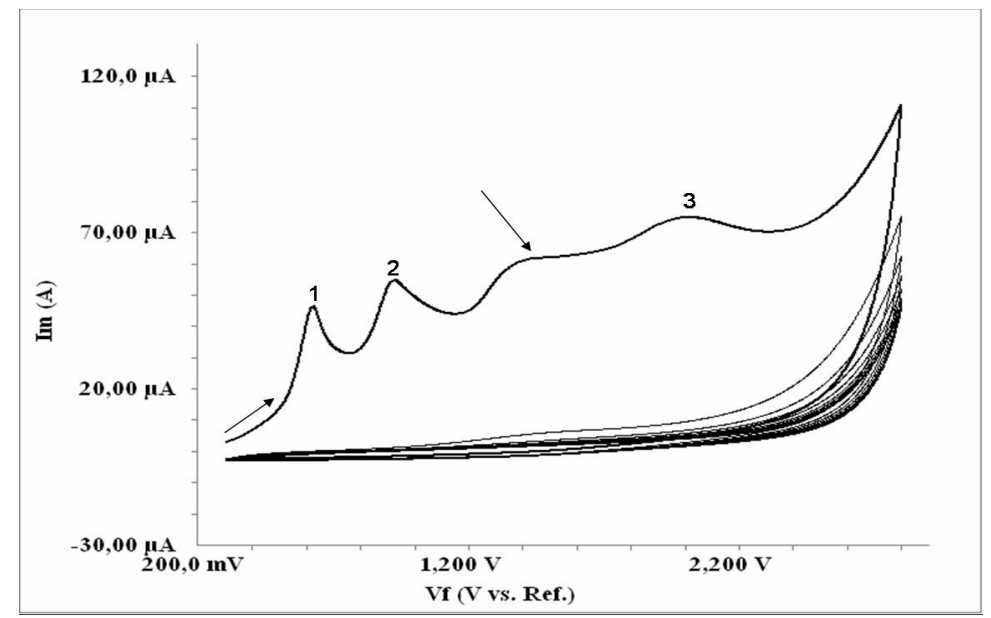

Figure 2. Cyclic voltammograms of quercetin on procaine modified glassy carbon electrode surface 
The characterization process following the modification process has been performed by using $\mathrm{CV}$ and EIS in non-aquous medium. For the $\mathrm{CV}$ characterization process, $1 \mathrm{mM}$ ferrocene solution in $0.1 \mathrm{M}$ TBATFB was used in the $-0.1 /+0.4 \mathrm{~V}$ potential range $100 \mathrm{mV} \mathrm{s}^{-1}$ scanning rate and it is shown in Figure 3(a). For the EIS characterization process, $1 \mathrm{mM}$ ferriyanide/ferrocyanide $(1: 1)$ solution mixture in $0.1 \mathrm{M} \mathrm{KCl}$ was used in the range of frequency $100000 / 0.05 \mathrm{~Hz}$ by impedance measurement and it is depicted in Figure 4. EIS is an effective method for probing the interfacial properties of modified electrodes and it also is a complementary technique to $\mathrm{CV}$ for evaluating the electrotransfer kinetics and the film compactness $^{31,32}$.

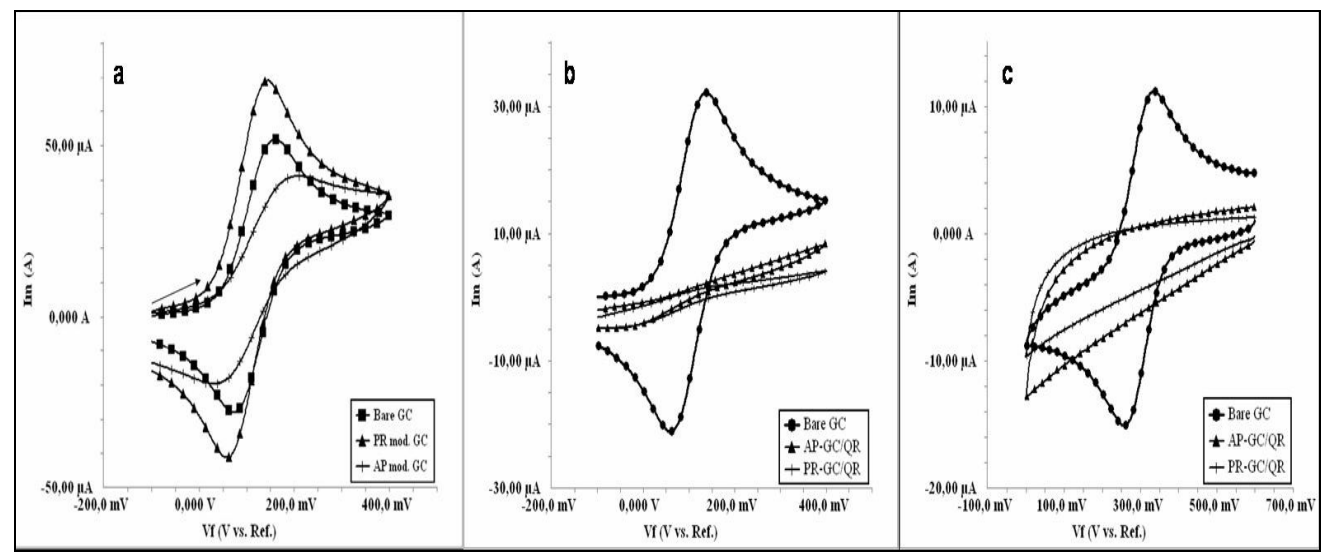

Figure 3. Surface voltammograms for QR modification (a) before modification with ferrocene (b) after modification with ferrocene (c) after modification with ferricyanide

The complex impedance can be presented as a sum of real, Zre, and imaginary, Zim, components that originate mainly from the resistance and capacitance of the cell, respectively. In the presence of $\mathrm{Fe}(\mathrm{CN})_{6}^{3-/ 4-}(1 \mathrm{mM})$ as the redox probe in $0,1 \mathrm{M} \mathrm{KCl}$ solution, the Randle's equivalent circuit is shown in Figure 4.

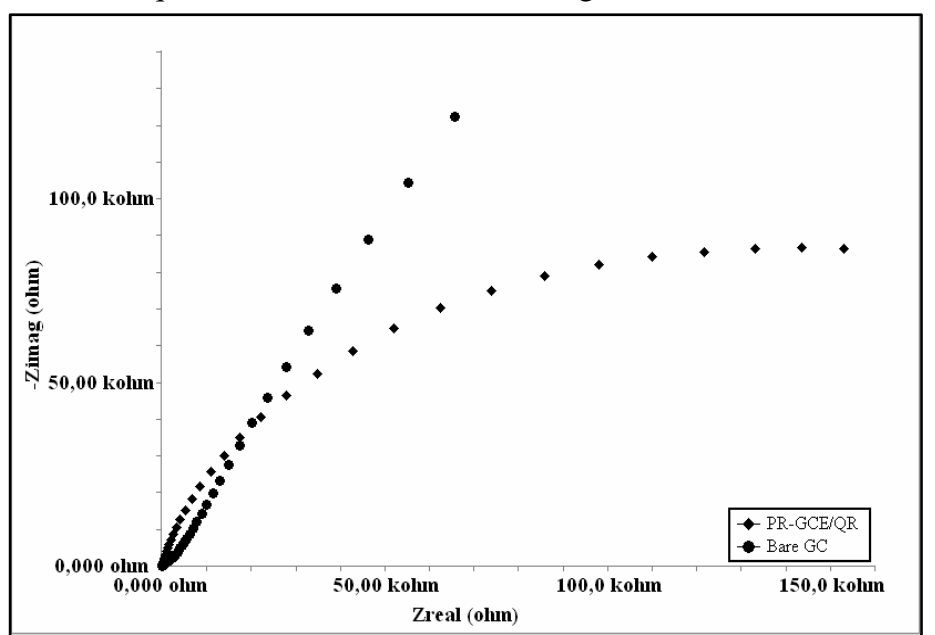

Figure 4. Nyquist graphs show that the electron transfer resistance for ferricyanide/ferrocyanide was increased by QR layer formed at the PR modified glassy carbon surfaces 


\section{Modification and characterization of quercetin on $A P-G C$}

The investigate the electrochemical behaviour of QR on aminophenyl modified GCE (APGCE) surface, NPDAS was synthesized starting from p-nitroaniline. For the modification process $1 \mathrm{mM}$ NPDAS solution (in 0.1 M TBATFB) was used in the $+0.2 /-0.8 \mathrm{~V}$ potential range using $100 \mathrm{mV} \mathrm{s}^{-1}$ scanning rate with 10 cycle (Figure 5).

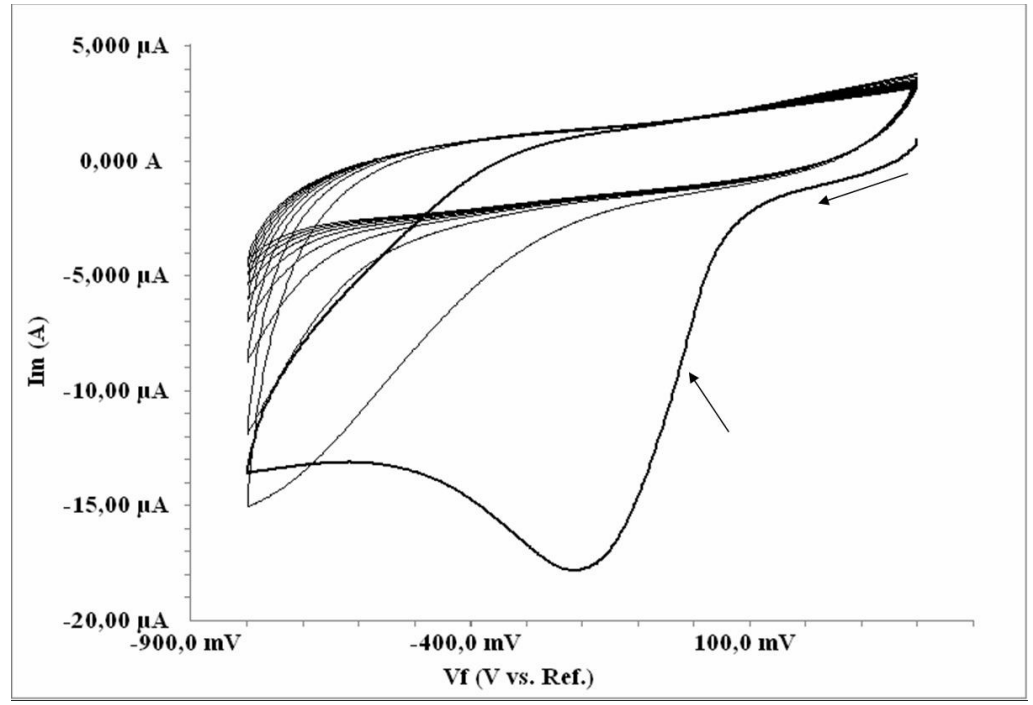

Figure 5. Cyclic voltammograms of NPDAS on bare glassy carbon electrode surface

The modified surface was electroinactive. So the surface was activated by reducing nitro group to amine group in the $-0.2 /-1.3 \mathrm{~V}$ potential range using $100 \mathrm{mV} \mathrm{s}^{-1}$ scanning rate with 5 cycle (Figure 6).

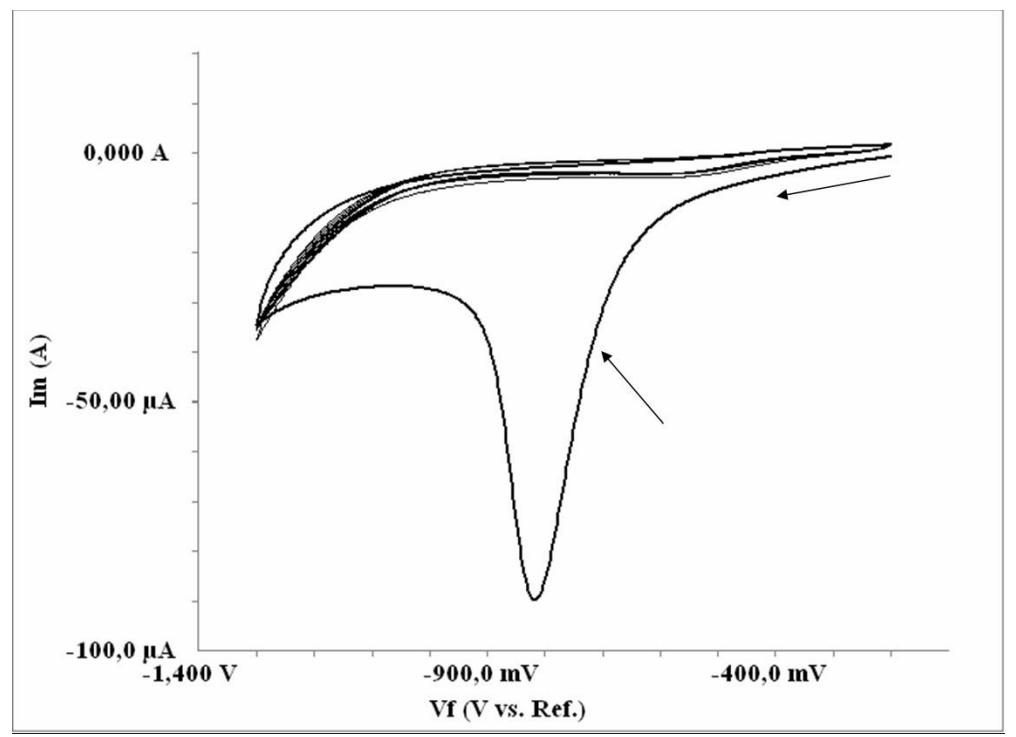

Figure 6. Reduction of nitro group voltammograms to amine on nitrophenyl modified glassy carbon electrode surface 
After the reduction of nitro group to amine group, AP-GCE surface was found to be electroactive from the modification and reduction process are given in Scheme 4(a) and 4(b).

a)

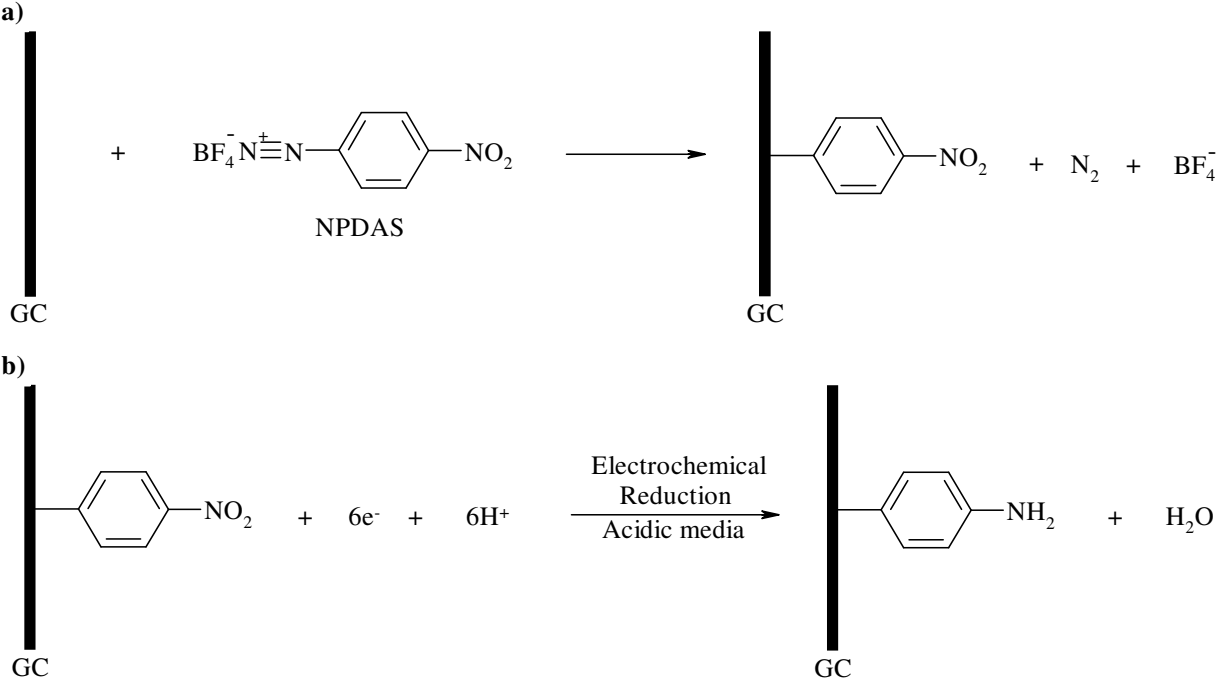

Scheme 4.(a) Modification of nitrophenyl group on glassy carbon electrode and (b) Reduction mechanism from nitro group into amine group electrode surface

The modification of QR on the obtained AP-GCE surface was carried out in the $+0.4 /+1.5$ $\mathrm{V}$ potential range using $100 \mathrm{mV} \mathrm{s}^{-1}$ scanning rate with 10 cycle and given in Figure 7.

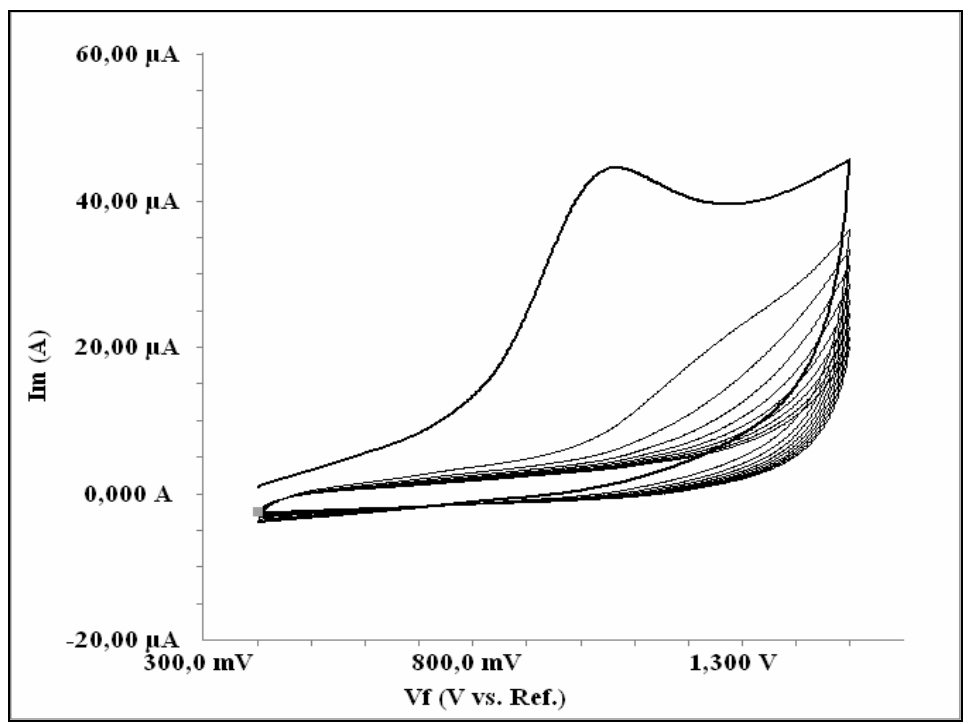

Figure 7. Cyclic voltammograms of quercetin on aminophenyl modified glassy carbon electrode surface

The surface characterization following the modification process was performed using $\mathrm{CV}$ in nonaquous and using EIS in aquous medium. $1 \mathrm{mM}$ Ferrocene solution (in $0.1 \mathrm{M}$ TBATFB) was used in the $-0.1 /+0.4 \mathrm{~V}$ potential range using $100 \mathrm{mV} \mathrm{s}^{-1}$ scanning rate for 
the characterization process done by $\mathrm{CV}$ (Figure $3 \mathrm{~b}$ ). $1 \mathrm{mM}$ Ferricyanide/ferrocyanide (1:1) mixture (in $0.1 \mathrm{M} \mathrm{KCl}$ ) was used in the $100000 / 0,05 \mathrm{~Hz}$ frequency range by measuring the impedance for the characterization process done by EIS (Figure 8).

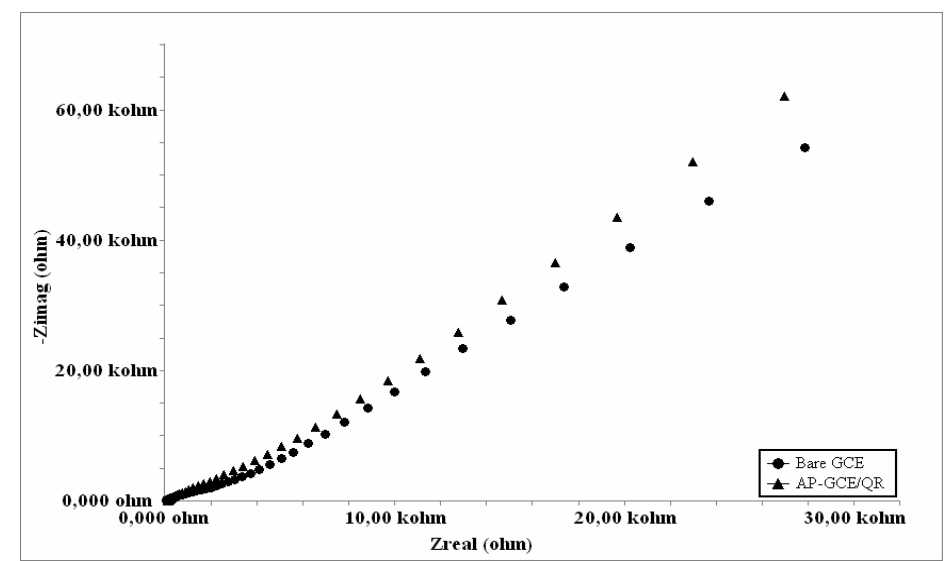

Figure 8. Nyquist graphs show that the electron transfer resistance for ferricyanide/ ferrocyanide was increased by QR layer formed at the AP modified glassy carbon surfaces

Reaction Mechanism for electrochemical modification of $Q R$ on PR-GCE and AP-GCE

According to the result, EC reaction mechanism follows the mechanism given in Scheme 5 during the modification of QR on the PR-GCE and AP-GCE surfaces. First QR molecule looses $3 \mathrm{e}^{-} / 3 \mathrm{H}^{+}$then the binding process take places. To understand the difference between the modification process, two voltammograms were overlayed and shown in Figure 9.

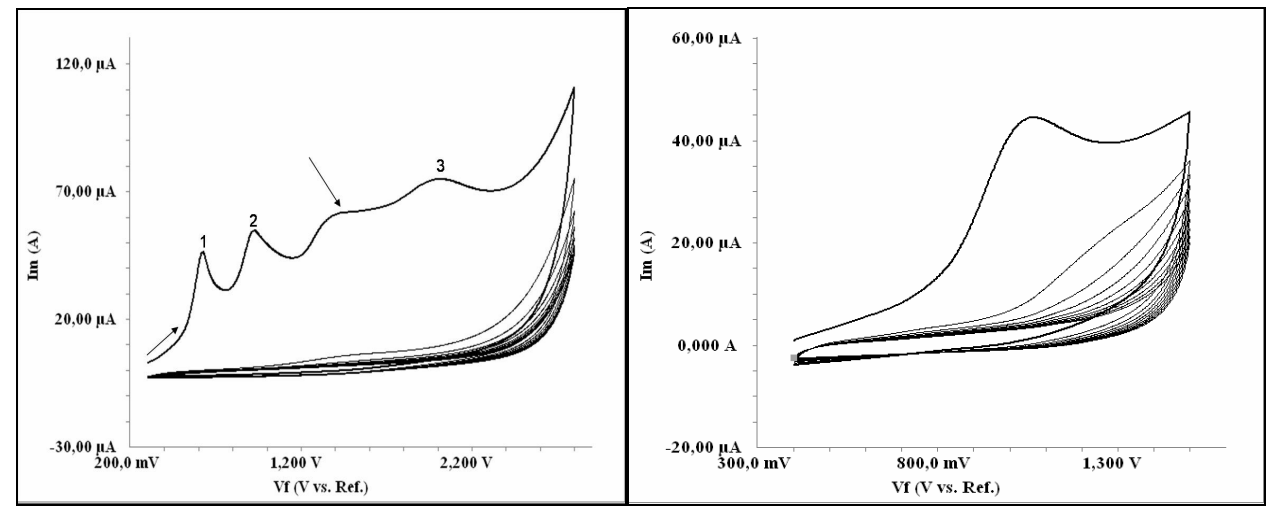

Figure 9. Cyclic voltammograms of quercetin on procaine and aminophenyl modified glassy carbon electrode surface

The first, second and third peaks are attributed to the $-\mathrm{OH}$ groups in the 3', 4' and 3 positions respectively. The binding of QR onto the PR-GCE and AP-GCE surfaces takes places through the $-\mathrm{OH}$ group in the position 3. Because -OH groups in the 3' and 4' positions were electrochemically oxidized forming o-quinone and become more stable ${ }^{21,23,30,33-36}$. Three different mechanisms were suggested for the binding of Quercetine to the procaine and aminophenyl modified GC surface. Based on the experimental results, the suggested mechanisms were explained by Michael addition. Quercetine in solution was first oxidized 
through the $-\mathrm{OH}$ groups in the 3' and 4' position. The oxidized form of quercetine, could bind covalently to the modified GC surface through three different positions in the form of N-C. Reaction mechanism was given in Scheme 5.

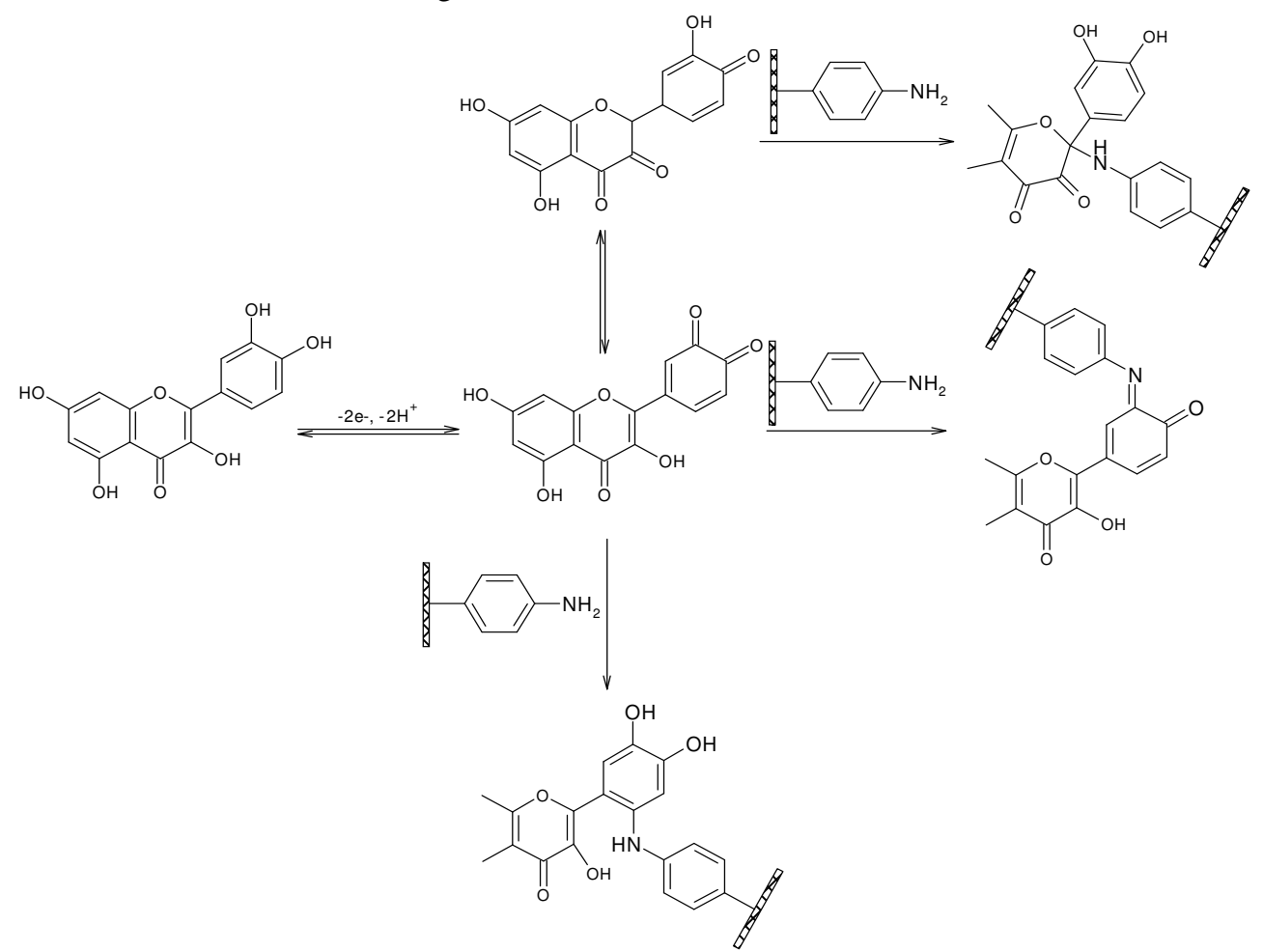

Scheme 5. Chemical binding of quercetine to the procaine and aminophenyl modified GC surface through Michael addition

\section{Conclusions}

In this research, the behaviour of quercetin was investigated by electrochemically and spectroscopically on the procaine and aminophenyl modified glassy carbon electrode surfaces. From the study, quercetin was found to binding through EC (Electrochemical and then Chemical) mechanism. Electrochemically oxidized quercetin at the first step binds to the electrode surface electrochemically at the second step. In the modification process of quercetin on the procaine and aminophenyl binded glassy carbon electrode surfaces, both electrochemical oxidation steps and chemical binding step were clearly determined. Based on the experimental results, a reaction mechanism was proposed and depicted in Scheme 5. The modification voltammograms obtained from cyclic voltammetry modification process, shows that quercetin binded strongly to the electrode surface. This result was supported by cyclic voltammetry surface test in non-aquous medium using ferrocene redox probe and by electrochemical impedance spectroscopy surface test in aquous medium using ferricyanide/ ferrocyanide redox probe mixture. The electroactive procaine and aminophenyl modified glassy carbon electrode surfaces become electroinactive after quercetin binding to these surfaces. As a result, electrochemical and spectroscopic measurements showed that bare glassy carbon electrode and quercetin modified electrode surfaces were different. 


\section{Acknowledgements}

This study was conducted as a part of Ph. D. Thesis of İbrahim Ender Mülazımoğlu. We would like to thank to the Prof. Ali Osman Solak (Ankara University, Science Faculty) and the Research Foundation of Selçuk University, Konya-TURKEY (BAP-06101020) for financial support of this work.

\section{References}

1. Strack D, Phenolic metabolism., In: Plant Biochemistry, Dey P.M, and Harbourne, Ed., Academic, San Diego, CA.,1997, 387.

2. Rice-Evans C A, Miller N J and Paganga G, Trends Plant Sci, 1997, 2, 152.

3. Larson R A, Phytochemistry, 1988, 27, 969.

4. Harborne J B and Williams C A, Phytochemistry, 2000, 55, 481.

5. Hotta H, Nagano S, Ueda M and Tsujino Y, Koyama J and Osakai T, Biochem. Biophys. Acta, 2002, 123, 1572.

6. Rice-Evans C A, Miller N J and Paganga G, Free Radical Biol. Med., 1996, 20, 933.

7. Heijnen C G M, Haenen G R M M, Vekemans J A J M and Bast, Environ. Toxicol. Pharmacol., 2001, 10, 199.

8. Yang B, Kotani A, Arai K and Kusu F, Anal. Sci, 2001, 17, 599.

9. Hotta H, Sakamoto H, Nagano S, Osakai T and Tsujino Y, Biochim. Biophys. Acta, 2001, 159, 1526.

10. Zuman P and Holthuis J J M, Recueil des Travaux Chimiques des Pays-Bas, 1988, 107, 403.

11. Janeiro P, Brett A M O, Electroanalysis, 2005, 17, 733.

12. Kikuzaki H, Hisamoto M, Hirose K, Akiyama K and Taniguchi H, J. Agric. Food Chem., 2002, 50, 2161.

13. Moon J H and Terao J, J. Agric. Food Chem., 1998, 46, 5062.

14. Thomas M J, Nutrition, 2000, 16, 716.

15. Wolf R, Wolf D and Ruocco V, J. Eur. Acad. Dermatol. Venereol., 1998, 10, 103.

16. Pietta P, J Nat. Prod., 2000, 63, 1035.

17. Rosenau T and Habicher W D, Tetrahedron, 1995, 51, 7919.

18. Hendrickson H. P, Kaufman A D and Lunte C E, J. Pharm. Biomed. Anal., 1994, 12, 325.

19. Yang B, Kotani A, Arai K and Kusu F, Anal. Sci., 2001, 17, 599.

20. Jorgensen L V, Cornett C, Justesen U, Skibsted L H and Dragsted L O, Free Radic. Res., 1998, 29, 339.

21. Brett A M O D and Malakzadeh M, J. Electroanal. Chem., 2003, 547, 191.

23. Timbola A K, De Souza C D, Giacomelli C and Spinelli A, J. Braz. Chem. Soc., 2006, 17, 139.

24. Korkina L G and Afanas'ev I B, Advances in Pharmacology, Academic Press Inc: New York, 1997, 151.

25. Rice-Evans C A and Packer L, Flavonoids in Health and Disease, Marcel Decker Inc: New York, 1998, 65.

26. Lemanska K, Szymusiak H, Tyrakowska B, Zielinski R, Soffers A E M F and Rietjens I M C M, Free Radical Biol. Med., 2001, 31, 869 p.

27. Janeiro P., Brett A. M. O., Anal. Chim. Acta, 2004, 518, 109 p.

28. İsbir A A, Solak A O, Üstündağ Z, Bilge S, Natsagdorj A, Kilic E and Kilic Z, Analytica Chimica Acta, 2005, 547, 59.

29. Solak A O, Eichorst L R, Clark W J and McCreery R L, Anal. Chem., 2003, 75, 296. 
30. Mulazimoglu I E, Ph. D. Thesis, 2008, Selçuk University, Institue of Natural and Applied Sciences, Konya, Turkey

31. Ren X and Pickup P G, J Electroanal Chem. 1997, 420, 251.

32. Patolsky F, Zayats M, Katz E and Willner I, Anal. Chem., 1999, 71, 3171.

33. Fardous A M, Abdel-Maaboud I M, Horria A M and Samiha A H, Talanta, 1996, 43, 1931.

34. Grotewold E., The Science of Flavonoids, 2006, Springer Science Business Media, Inc., Department of Cellular and Molecular Biology, The Ohio State University, Columbus, Ohio,

35. Andersen Q M, Markham K R, Flavonoids: Chemistry, Biochemistry and Applications, 2006, LLC CRC Press is an imprint of Taylor \& Francis Group

36. Zare H R, Namazian M and Nasirizadeh N, J Electroanal. Chem., 2005, 584, 77. 


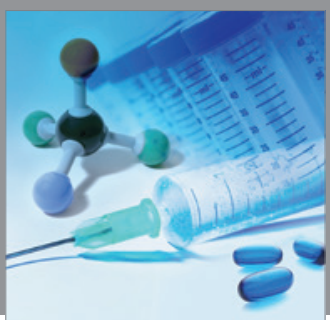

International Journal of

Medicinal Chemistry

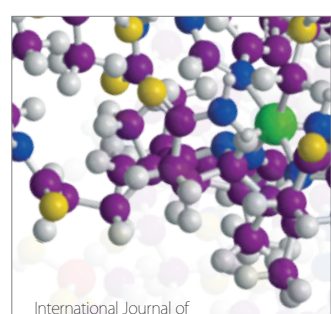

Carbohydrate Chemistry

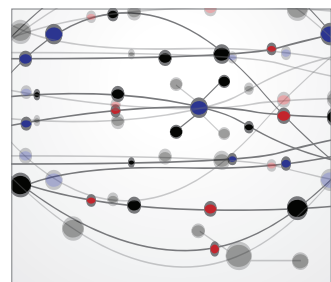

The Scientific World Journal
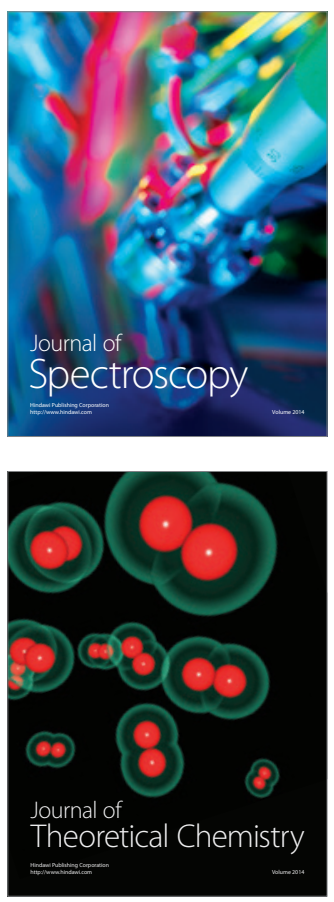
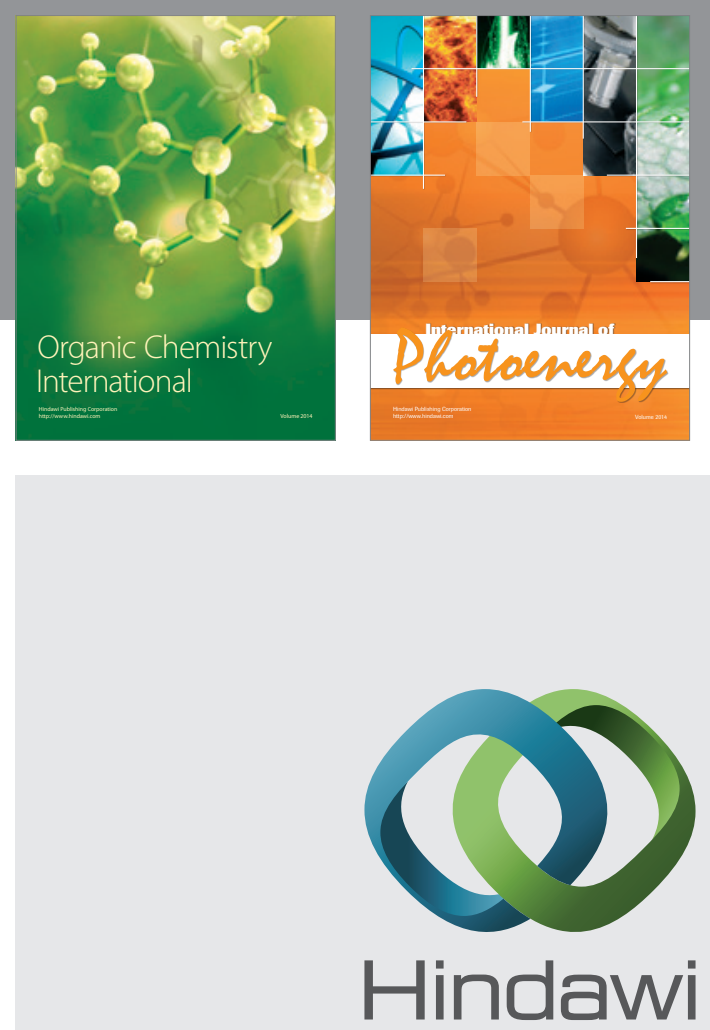

Submit your manuscripts at

http://www.hindawi.com
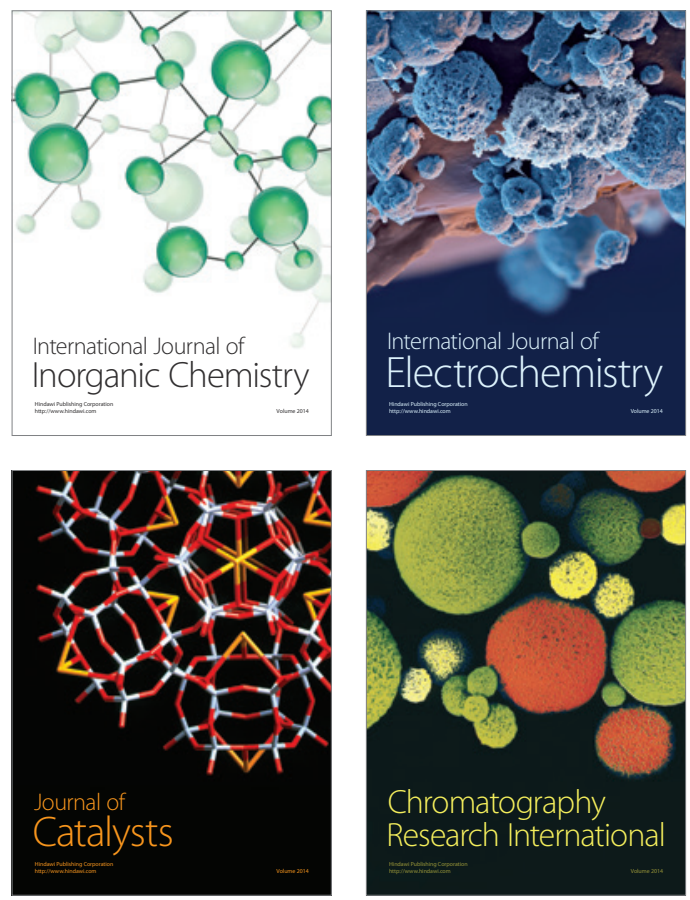
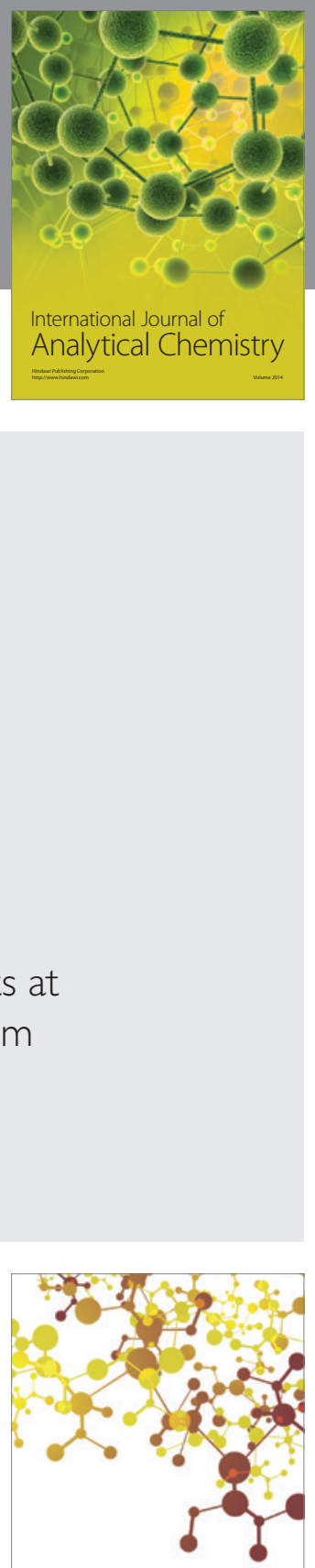

Journal of

Applied Chemistry
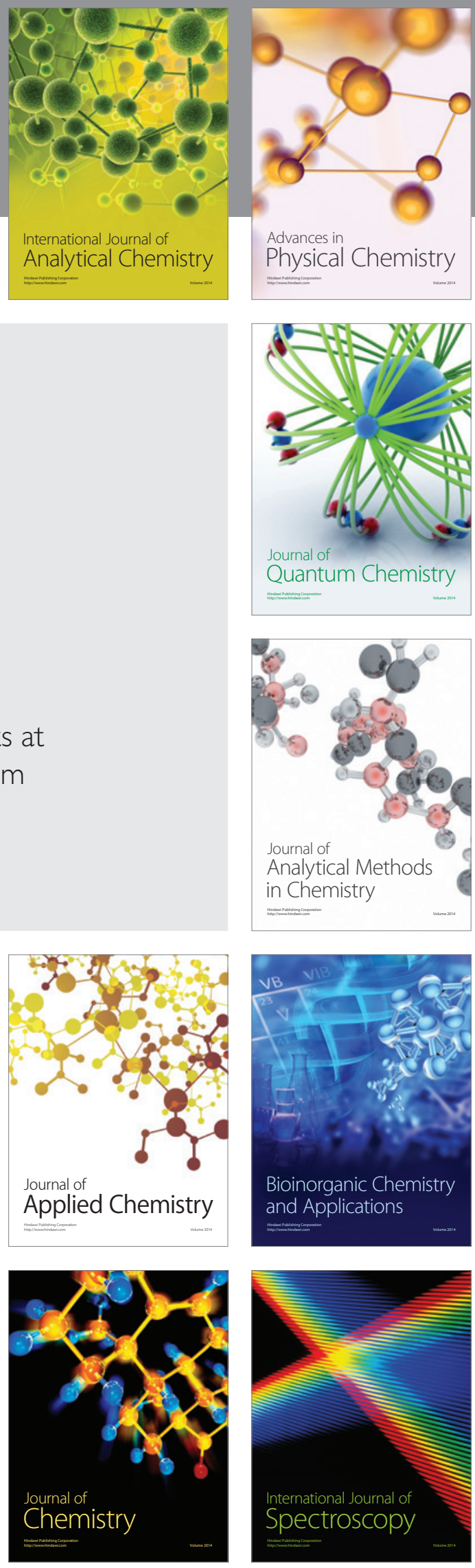